\title{
Young Stellar Populations in M31, M33, and NGC 6822
}

\author{
J.B. Hutchings, B. Cavanagh \\ DAO/HIA/NRC, Victoria, Canada
}

L. Bianchi, R. Chandar

Johns Hopkins University, Baltimore, MD, USA

S. Scuderi

Catania Observatory, Catania, Italy

\begin{abstract}
.
We have been pursuing a program of hot star UV spectroscopy in local group galaxies over several years with HST. We have also obtained WFPC2 images in parallel and pointed exposures with several broad band filters.

- The imaging data have been used to generate colour-magnitude plots of central fields, clusters and halo fields in M31, M33 and NGC 6822. We have compared these with high-mass model isochrones from recent models, to examine the stellar populations that are indicated. M33 contains a substantial young cluster population whose mass and age appear to be related. The western outer wisps of NGC 6822 contain a relatively young population of stars.

- Most of the spectra have been reported and modelled in published papers: we note here the first comparison of stars in NGC 6822 with others from local group galaxies.

- We have also discussed the interstellar extinction in M31 and find it is similar to the Galaxy after allowance for comparable amounts of halo extinction that resembles that of the Magellanic Clouds.
\end{abstract}

\section{NGC 6822}

We have covered two regions in the outer parts of this galaxy with multicolour imaging. The NE region is slightly further away, while the $\mathrm{W}$ region lies within the faint low surface brightness outer regions that are present on that side of the galaxy.

The $W$ region contains a main sequence that extends to stars of about $2 M_{\odot}$, with an age of about $200 \mathrm{Myr}$. The NE region has no main sequence or stars younger than $1 \mathrm{Gyr}$, but does contain some luminous red stars that are not matched in the $\mathrm{W}$ field. These stars are not clumped in the field. The upper plot in Fig. 1 shows a colour-magnitude diagram of the outer fields which has 
been foreground-subtracted using images from a different high latitude field from the HST archive, which had similar exposures in two of our filters. Isochrones derived from Bertelli et al (1994) are superposed.

We also show a colour-magnitude diagram for one WFPC2 chip containing the young star-forming region Hubble V in NGC 6822, derived from our fourcolour imaging. The measured stellar magnitudes have been dereddened estimating the extinction from the reddening-free $Q$ parameter for each star. The comparison with isochrones indicates the presence of several stars only a few million years old. Global comparison with the other colours available indicates a possibly non-standard reddening law in the star-forming regions.

\section{M31 and M33 Fields}

We have images in F555W, F300W, and F160W in M31. The UV data are not useful for population studies as they were single exposures which do not allow cosmic ray removal. Also, there are very few stars and the detectors have a significant red leak. The other two filters produced useful colour-magnitude diagrams. The models for these colours indicate a population some $60 \mathrm{Myr}$ old.

One of the M31 field CCDs lies in a dust lane and contains a very different distribution of stars in the HR diagram. The bulk of the faint stars is missing, presumably reddened beyond detectability in the F300W filter. The stars that are detected lie outside the dust lanes, but there are fewer faint stars detected over the whole region. The brighter stars have a similar colours to those seen in the other CCDs, and are presumably not appreciably reddened.

In our M33 diagrams, different populations (young massive stars from OB associations, and older field stars) are present. The measured photometry in these images was corrected for the colour terms, so $U B V$ magnitudes are not instrumental, but classical $U B V$. No dereddening was applied to the data points, and no transformation to $T_{e f f}$ and $L_{b o l}$, because for very massive stars the $U-B, B-V$ colors are not sensitive enough to their high temperatures, so the transformation would introduce significant errors. The NGC 6822 and M31 data will be published in full elsewhere.

\section{Clusters in M33}

Star clusters are a useful tool in the analysis of the stellar populations of galaxies, as they represent single age and abundance points which can be used to trace star-formation activity. In five HST WFPC2 fields in M33 from our program GO6038 we discovered 60 stellar clusters (49 of which have not been previously catalogued). The $U B V$ and far UV filters, selected for the purpose of studying the hot massive star population, are also ideal for studying young star clusters, and the HST resolution allowed us to observe crowded nuclear and spiral arm regions which have not been studied to date. The cluster characteristics range from UV-bright young clusters to red globular clusters. Most of the objects in our sample, however, have morphologies consistent with the 'populous clusters' abundant in the Magellanic Clouds, and absent in the Milky Way and M31.

The integrated $(B-V)$ vs. $(U-B)$ cluster colours have been compared with stellar evolution models to estimate cluster ages. We find the star clusters 

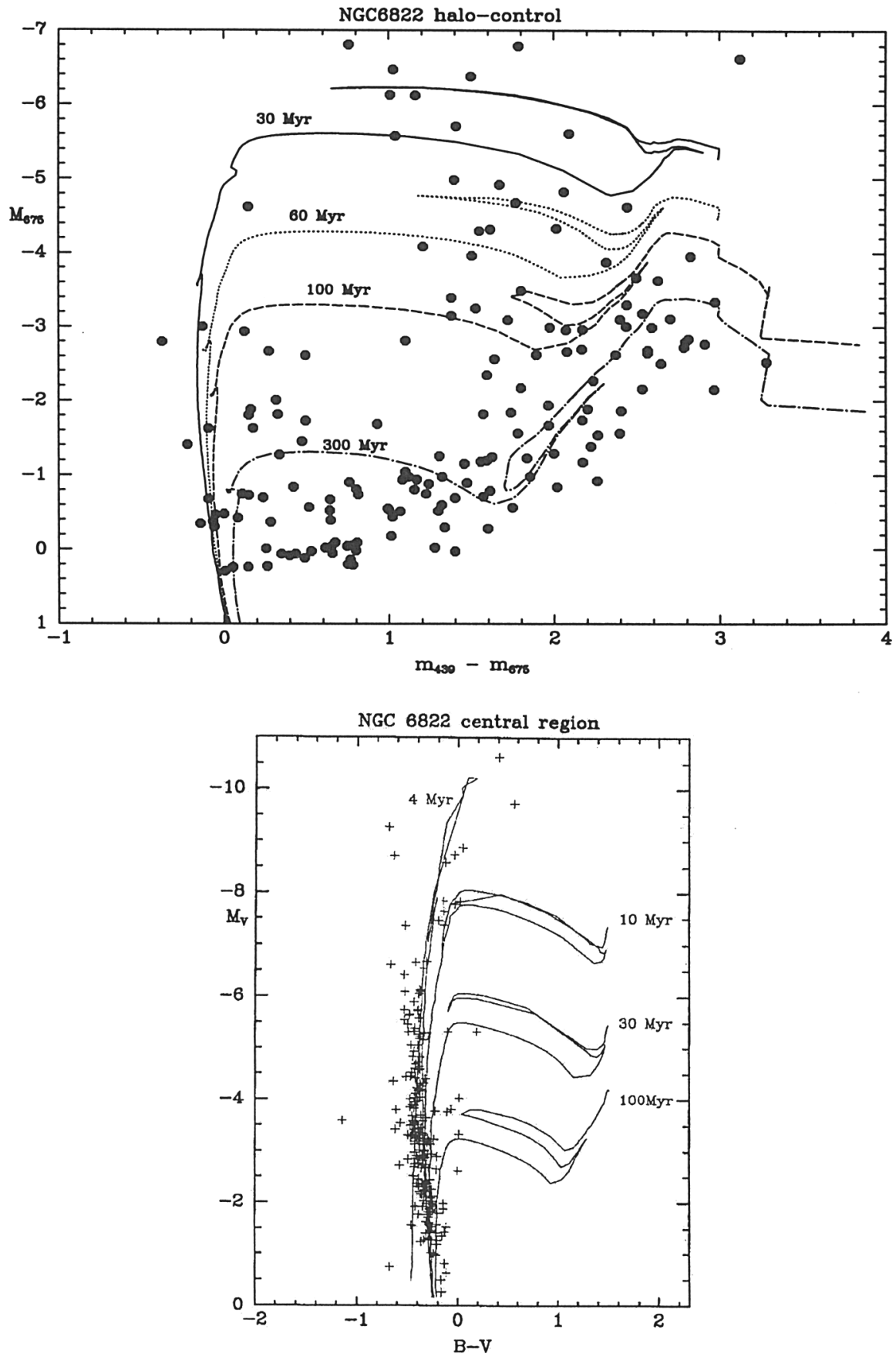

Figure 1. NGC 6822 CM plots. Upper: outer fields corrected for foreground stars. Lower: H II region Hubble V. 
to have a range of ages from $4 \times 10^{6}$ to $10 \times 10^{9} \mathrm{yrs}$, and that formation of these clusters has been fairly continuous during this period. Luminosities and ages combined with theoretical mass-to-light ratios give mass estimates for M33 star clusters between $4 \times 10^{2}-3 \times 10^{5} M_{\odot}$. An important result is that M33 cluster masses have been systematically decreasing from the oldest to youngest (from Chandar, Bianchi \& Ford 1998).

\section{UV Spectroscopy}

Some of the recent results can be summarized as follows:

1. Wind velocities and mass loss rates of M31 stars are comparable to Galactic values (Bianchi et al. 1996a, Bianchi 1998).

2. Chemical peculiarities are revealed by the UV spectra of some M31 stars e.g. $\mathrm{C}$ or $\mathrm{N}$ significantly underabundant in stars having solar-like abundances of other elements (Bianchi et al. 1996a).

3. The UV extinction curve in M31 is similar to the Galactic average extinction curve in slope, but the $2175 \AA$ feature is possibly weaker (Bianchi et al. 1996b).

4. Wind velocities and UV line intensities in M33 hot supergiants are comparable to LMC counterparts and weaker than in Galactic and M31 counterparts (Bianchi et al. 1996a; Bianchi 1998).

5. Narrow absorption components are detected in an M33 object, confirming clumpiness of the mass outflow to be a ubiquitous phenomenon (Bianchi et al. 1996a)

6. Measured stellar parameters and the wind momentum rate agree with theoretical models (Bianchi et al. in preparation)

7. Hot stars in NGC 6822 reveal similarities to SMC objects in their UV lines, as expected for the extremely low metallicity. However, one object shows Galacticlike metallicity and wind velocity. Further investigation in this region of the galaxy is under way, to determine whether a strong metallicity gradient exists (Scuderi \& Bianchi 1997).

\section{References}

Bertelli, G., Bressan, A., Chiosi, C., Fagotto, F., Nasi, E. 1994, A\&AS, 106, 275 Bianchi, L., Hutchings, J.B., Massey, P. 1996a, AJ, 111, 2303

Bianchi, L., Bohlin, R., Clayton, G., Hutchings, J., Massey, P. 1996b, BAAS, 187,4511

Bianchi, L. 1998, ESA SP-413, p. 553

Chandar, R., Bianchi, L., Ford, H. 1998, A\&AS, submitted

Scuderi, S., Bianchi, L. 1997, BAAS, 29, 806

\section{Discussion}

van den Bergh: Could the apparent correlation that you find between the ages and the luminosities of clusters in M33 be due to the fact that only the most 
massive clusters survive for a long time?

Hutchings: The most massive clusters are the oldest, so while we may have lost low mass old clusters, we still find no high mass young clusters. Thus the relationship may indicate a decreasing upper limit of mass with time - possibly steeper than shown if mass is being lost. 